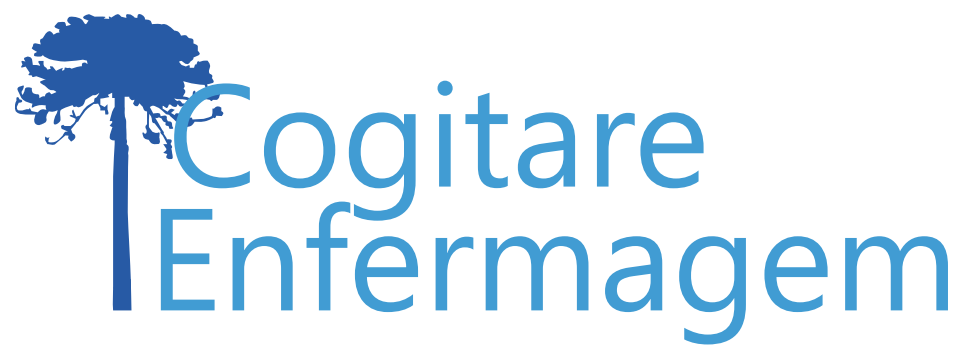

\title{
SÍNDROME DE BURNOUT ENTRE PROFISSIONAIS DE UM SERVIÇO DE ATENDIMENTO MÓVEL DE URGÊNCIA DO PARANÁ
}

Gabriela Bettoni Figueiroa1, Hellen Emília Peruzzo², Nelly Lopes Moraes Gil ${ }^{3}$, Ivi Ribeiro Back ${ }^{4}$, Eraldo Schunk da Silva ${ }^{5}$, Sonia Silva Marcon ${ }^{6}$

\section{RESUMO}

Objetivo: identificar o risco para o desenvolvimento da Síndrome de Burnout em profissionais do Serviço de Atendimento Movel de Urgência (SAMU).

Metodologia: estudo transversal, não probabilístico, realizado entre agosto e outubro de $2017 \mathrm{com}$ 101 profissionais atuantes em um SAMU do Paraná, utilizando questionário autoaplicável - Maslach Burnout Inventory. A análise de dados envolveu os testes de associação Qui-quadrado, Wilcoxon e Mann-Whitney.

Resultados: observa-se que o risco para desenvolvimento e manifestação da síndrome de Burnout foi identificado em 52 profissionais $(51,9 \%$ ) e foi mais frequente entre médicos e teleatendentes, indivíduos do sexo feminino, com mais de 40 anos, sem companheiro, sem filhos, que tinham outro vínculo empregatício e renda superior a 10 salários mínimos. Porém, só os que trabalhavam durante o dia apresentaram risco significativamente maior $(p=0,0075)$. Conclusão: as características do processo de trabalho no SAMU geram sobrecarga aos profissionais, sendo importante a implementação de estratégias que possam minimizar os efeitos estressores desta atuação.

DESCRITORES: Unidades Móveis de Saúde; Esgotamento Profissional; Pessoal de Saúde; Despersonalização; Ambiente de trabalho.

COMO REFERENCIAR ESTE ARTIGO:

Figueiroa GB, Peruzzo HE, Gil NLM, Back IR, Silva ES da, Marcon SS. Síndrome de burnout entre profissionais de um serviço de atendimento móvel de urgência do Paraná. Cogitare enferm. [Internet]. 2019 [acesso em "colocar data de acesso, dia, mês abreviado e ano"]; 24. Disponível em: http://dx.doi.org/10.5380/ce.v24i0.61917.

\section{(c) (1)}

Este obra está licenciado com uma Licença Creative Commons Atribuição 4.0 Internacional.

${ }^{1}$ Enfermeira. Residente Intensivista da Pontifícia Católica de Campinas. Campinas, SP, Brasil.

${ }^{2}$ Enfermeira. Doutoranda em Enfermagem. Universidade Estadual de Maringá. Maringá, PR, Brasil. ()

${ }^{3}$ Enfermeira. Doutora em Enfermagem. Docente de Enfermagem da Universidade Estadual de Maringá. Maringá, PR,

Brasil. 1

${ }^{4}$ Enfermeira, Nutricionista. Pós-doutoranda em Enfermagem na Universidade Estadual de Maringá. Maringá, PR, Brasil. (-)

${ }^{5}$ Estatítico. Doutor em Estatística. Docente da Universidade Estadual de Maringá. Maringá, PR, Brasil. $\bigcirc$

${ }^{6}$ Enfermeira. Doutora em Filosofia da Enfermagem. Docente de Enfermagem da Universidade Estadual de Maringá. Maringá, PR, Brasil. (2) 


\title{
BURNOUT SYNDROME IN PROFESSIONALS OF A MOBILE EMERGENCY SERVICE IN THE STATE OF PARANÁ
}

\begin{abstract}
Objective: To identify the risk for the development of Burnout Syndrome in professionals of the Mobile Emergency Care Service (SAMU).

Methodology: Cross-sectional non-probabilistic study conducted between August and October 2017 with 101 professionals of a Mobile Emergency Care Service (SAMU) of the State of Paraná, through the self-administered questionnaire - Maslach Burnout Inventory. Data analysis was performed using Chi-square, Wilcoxon and Mann-Whitney association tests. Results: The risk for the development and manifestation of Burnout syndrome was identified in 52 professionals (51.9\%) and was more frequent among physicians and telephone operators, females over 40 years old, without partners, without children, who had another employment and income of more than 10 minimum wages. However, only those who worked in the day shift were at a significantly higher risk for the syndrome $(p=0.0075)$.

Conclusion: The daily process of work at the SAMU mobile emergency generates overload of work to health professionals. Therefore, strategies aimed to minimize the stressful effects of their work activities should be implemented.
\end{abstract}

DESCRIPTORS: Mobile Health Units; Professional Exhaustion; Health Personnel; Depersonalization; Workplace.

\section{SÍNDROME DE BURNOUT ENTRE PROFESIONALES DE UN SERVICIO DE ATENDIMIENTO MOVIL DE URGENCIA DE PARANÁ}

\author{
RESUMEN \\ Objetivo: identificar el riesgo para el desarrollo del Síndrome de Burnout en profesionales del \\ Servicio de Atendimiento Móvil de Urgencia (SAMU). \\ Metodología: estudio trasversal, no probabilístico, que se realizó entre agosto y octubre de \\ 2017 con 101 profesionales que actúan en un SAMU de Paraná, utilizando cuestionario auto \\ aplicable - Maslach Burnout Inventory. El análisis de datos abarcó las pruebas de asociación \\ Chi cuadrado, Wilcoxony Mann-Whitney. \\ Resultados: se identificó riesgo para desarrollo y manifestación del síndrome de Burnout en \\ 52 profesionales $(51,9 \%)$, siendo éste más frecuente entre médicos y profesionales que ope- \\ ran el teléfono, individuos del sexo femenino, con más de 40 años, sin pareja, sin hijos, que \\ tenían otro vínculo laboral y renta superior a 10 sueldos mínimos. Sin embargo, solamente \\ aquellas personas que trabajaban durante el día presentaron riesgo significativamente mayor \\ $(p=0,0075)$. \\ Conclusión: las características del proceso de trabajo en SAMU generan sobrecarga a los \\ profesionales, siendo importante la implementación de estrategias que puedan minimizar los \\ efectos estresores de esta actuación.
}

DESCRIPTORES: Unidades Móviles de Salud; Agotamiento Profesional; Equipo de Salud; Despersonalización; Ambiente de trabajo. 
Burnout é uma síndrome que surge em resposta à exposição crônica ao estresse no ambiente de trabalho(1-2). Sua manifestação envolve três componentes relacionados, porém independentes: a) exaustão emocional, que se refere à falta de energia, entusiasmo e sentimento de esgotamento; b) despersonalização, que se caracteriza pelo comportamento negativo do profissional ao lidar com clientes, colegas de trabalho e chefia como objetos; e c) redução da realização pessoal, caracterizada por autoavaliação negativa do trabalhador em relação ao seu desenvolvimento profissional(3).

Entre os profissionais da área da saúde, a manifestação da síndrome de Burnout é bastante frequente. Isto se deve à própria natureza do trabalho nesta área, em que os profissionais geralmente desenvolvem uma série de atividades que exigem controle emocional e mental, expondo-os constantemente a situações de estresse. Dado a estas características, a síndrome de Burnout acomete de forma mais frequente os profissionais que realizam o cuidado de forma direta, contínua e que estão emocionalmente envolvidos com o paciente ${ }^{(1,2)}$.

A associação de fatores diversos presentes no ambiente de trabalho potencializa o risco de desenvolver Síndrome de Burnout $t^{(4,5)}$. Os integrantes das equipes de enfermagem, por exemplo, são constantemente expostos a esgotamento profissional e estresse no local de trabalho, constituindo, portanto, um grupo de grande risco ao desenvolvimento do Burnout. Isto ocorre porque, além de permanecem mais tempo ao lado do paciente e de seus familiares, também convivem com a tensão diária de ter que cuidar de uma vida e necessitar tomar decisões relacionadas a estes cuidados ${ }^{(1,4)}$. A dupla jornada de trabalho também é bastante frequente entre eles, principalmente em decorrência da necessidade (pessoal ou do serviço) de realizar horas extras, inclusive para suprir demandas relacionadas ao absenteísmo ou afastamentos por motivos de saúde.

É importante destacar que os profissionais que atuam no Serviço de Atendimento Móvel de Urgência (SAMU) também estão diária e frequentemente envolvidos com situações estressantes. Este serviço é o componente pré-hospitalar móvel da Política Nacional de Atenção às Urgências, que, por meio do atendimento a vítimas no local da ocorrência, tem por objetivo reduzir o número de óbitos, o tempo de internação e as complicações decorrentes da falta de atendimento imediato ${ }^{(6)}$.

O SAMU funciona de maneira ininterrupta e realiza atendimentos em residências, locais de trabalho e vias públicas, a partir de chamadas gratuitas, em todo território nacional, para urgências médicas, no número 192. O atendimento é realizado por equipe multiprofissional, cuja composição depende do nível de complexidade da ocorrência. A equipe de suporte básico de vida é constituída por condutor socorrista e auxiliar de enfermagem e destina-se ao transporte pré e inter-hospitalar de pacientes sem risco de vida. Já a equipe de Suporte Avançado (SAV) é constituída por condutor socorrista, enfermeiro, médico e auxiliar de enfermagem. Destina-se ao atendimento e transporte pré-hospitalar de pacientes em situação de emergência ou transporte inter-hospitalar dos que precisam de cuidados médicos intensivos ${ }^{(6,7)}$.

Segundo pesquisa realizada sobre saúde e qualidade de vida de profissionais do SAMU, grande parte dos atendimentos realizados envolve causas externas e condições clínicas complexas que requerem habilidade e competência técnica para avaliação minuciosa e decisão qualificada. Destarte, as decisões tomadas em relação aos primeiros atendimentos e encaminhamentos são essenciais na manutenção das condições vitais e para diminuir e/ou evitar agravamento do quadro clínico e/ou sequelas ${ }^{(8)}$.

Apesar dos profissionais que atuam neste serviço receberem capacitações técnicas específicas e existirem protocolos para o manejo apropriado de pacientes em situação de agravo clínico e/ou traumático, na prática eles enfrentam diferentes e concomitantes situações estressantes. De acordo com pesquisa realizada em São Paulo, estes profissionais carecem de maior preparo para compartilharem com o enfermo e seus familiares sentimentos 
como angústia, dor e medo da morte, os quais influenciam sua atuação e seu processo de trabalho(4).

Isto posto, definiu-se como objetivo do estudo identificar o risco para o desenvolvimento da Síndrome Burnout entre profissionais do Serviço de Atendimento Móvel de Urgência de um município do Paraná.

\section{MÉTODO}

Estudo transversal realizado no SAMU de um município do noroeste do Paraná, o qual atende 30 municípios. À época do estudo, contava com 13 ambulâncias, sendo três delas de Suporte Avançado de Vida (SAV) e 10 de Suporte Básico de Vida.

Os dados foram coletados no período de agosto a outubro de 2017. Fizeram parte do estudo 101 profissionais médicos, condutores socorristas, enfermeiros, teleatendentes e auxiliares de enfermagem, de um universo de 185 atuantes no serviço. Os participantes foram selecionados por conveniência e foram abordados durante o turno de trabalho, nas dependências do serviço.

Constituíram critérios de inclusão: ser médico, enfermeiro, teleatendente, condutor socorrista ou auxiliar/técnico de enfermagem e atuar no SAMU há pelo menos seis meses. Os critérios de exclusão foram: estar em licença médica ou férias e não estar de serviço nos dias destinados à coleta de dados.

Foram convidados a participar do estudo todos os profissionais que atendiam aos critérios de inclusão previamente definidos, localizados durante o período da coleta de dados. No total foram realizadas 32 visitas ao serviço, em dias e turnos diferentes. Novas visitas foram realizadas até que a maioria dos profissionais atuantes no serviço tivesse sido contatada. Dos 185 profissionais elegíveis para o estudo, cinco se recusaram a participar, 14 estavam afastados (licença médica ou férias), 17 atuavam no serviço há menos de seis meses e os demais não foram encontrados, seja por estarem em atendimento externo ou por incompatibilidade com a escala de serviço. Ressalta-se que alguns médicos eram contratados como prestadores de serviço, o que justifica a dificuldade de serem encontrados, pois possuem escala de serviço diferenciada, sem carga horária pré-definida.

Os dados foram coletados por meio de questionário autoaplicável, constituído de duas partes, a primeira, elaborada pelos autores, abordando características sóciodemográficas (sexo, idade, estado civil, categoria profissional, outro vínculo empregatício, turno de trabalho, renda familiar e número de filhos) e a segunda, constituída pelo Maslach Burnout Inventory - Human Services Survey (MBI-HSS).

O MBI-HSS foi elaborado por Christina Maslach e Susan Jackson em 1996 e traduzido/ validado para o português, ${ }^{(3)}$ e se propõe a identificar as dimensões sintomatológicas da síndrome de Burnout. E constituído por 22 questões, nove destinadas à identificação do o nível de Exaustão emocional (falta de energia, entusiasmo e sentimento de esgotamento), oito à Realização pessoal (autoavaliação do trabalhador) e cinco à Despersonalização (comportamento profissional negativo). As respostas são apresentadas em escala do tipo likert de seis pontos $(0=$ nunca; $1=$ algumas vezes no ano; 2 = uma vez ao mês; $3=$ algumas vezes no mês; $4=$ uma vez por semana; $5=$ algumas vezes por semana; e $6=$ todos os dias).

O escore em cada uma das três dimensões é calculado pela somatória dos pontos dos itens relativos a cada um dos domínios. Pontuações elevadas em relação à "Exaustão emocional" (entre 26 a 54 pontos) e "Despersonalização" (entre 09 a 30 pontos), associadas à baixa pontuação na "Realização pessoal" (0 a 33 pontos), podem indicar risco para a manifestação da síndrome de Burnout ${ }^{(3)}$, conforme Quadro 1. 
Quadro 1 - Domínios da escala do MBI para classificação de Síndrome de Burnout, 2011. Maringá, PR, Brasil, 2017

\begin{tabular}{|l|c|c|c|}
\hline \multirow{2}{*}{ Dimensões da escala } & \multicolumn{3}{|c|}{ Pontuações } \\
\cline { 2 - 4 } & Baixa & Média & Elevada \\
\hline Exaustão emocional & $0-15$ & $16-25$ & $\underline{26-54}$ \\
\hline Despersonalização & $0-02$ & $03-08$ & $\underline{09-30}$ \\
\hline Realização pessoal & $\underline{0-33}$ & $34-42$ & $43-48$ \\
\hline
\end{tabular}

Fonte: Trigo, 2011(3).

Para comparar a igualdade entre os diferentes níveis das variáveis de controle e os componentes de diagnóstico da síndrome de Burnout, utilizou-se o Teste de Associação Qui-quadadro e de Wilcoxon-Mann-Whitney, com aproximação para a Distribuição Normal (Z) ou o teste de Kruskal-Wallis (KW). Todos os testes foram unicaudais e para todos considerou-se um nível de confiança de $95 \%(\alpha=0,05)$, ou seja, $p$-valor menor que 0,05. Os dados foram analisados no programa Statistical Analysis Software, a partir de uma base de dados construída no aplicativo Excel.

O projeto foi aprovado pelo Comitê Permanente de Ética em Pesquisas com Seres Humanos, $n^{\circ} 2.230 .015$ de uma universidade pública brasileira.

\section{RESULTADOS}

Dos 101 profissionais em estudo, a maior proporção foi de médicos, seguido pelos condutores socorristas, enfermeiros, teleatendentes e auxiliares de enfermagem (Tabela 1). Porém, ao se avaliar a proporção de participantes dentro de cada categoria, observouse que as maiores participações por ordem de frequência foram de enfermeiros $(90,5 \%)$, teleatendentes $(84,2 \%)$, condutores socorristas $(71 \%)$, auxiliares de enfermagem $(58,3 \%)$ e médicos (42,2\%). A baixa participação dos médicos é justificada pelo fato de que estes profissionais atuam como prestadores de serviços e por isto possuem escala de trabalho bastante flexível.

Tabela 1 - Risco de desenvolver a manifestação da síndrome de Burnout segundo variáveis sóciodemográficas e profissionais. Maringá, PR, Brasil, 2017 (continua)

\begin{tabular}{|c|c|c|c|c|}
\hline \multirow{2}{*}{ Variáveis } & \multicolumn{2}{|r|}{$\begin{array}{c}\text { Risco para } \\
\text { Burnout } \\
\text { (n=52) } \\
\end{array}$} & \multirow{2}{*}{$\begin{array}{c}\begin{array}{c}\text { Sem risco } \\
\text { para Burnout } \\
(n=49)\end{array} \\
\begin{array}{c}\text { N (Escore } \\
\text { Médio) }\end{array}\end{array}$} & \multirow[t]{2}{*}{ p-valor } \\
\hline & $\mathbf{n}$ & $\begin{array}{c}\text { N (Escore } \\
\text { Médio) }\end{array}$ & & \\
\hline \multicolumn{5}{|l|}{ Sexo } \\
\hline Masculino & 55 & $26(47,3)$ & $29(52,7)$ & \multirow[t]{2}{*}{0,1039} \\
\hline Feminino & 46 & $26(56,5)$ & $20(43,5)$ & \\
\hline \multicolumn{5}{|l|}{ Faixa Etária } \\
\hline Menos de 40 anos & 62 & $34(54,8)$ & $28(45,2)$ & \multirow[t]{2}{*}{0,0764} \\
\hline 40 anos ou mais & 39 & $15(41,7)$ & $21(58,3)$ & \\
\hline
\end{tabular}




\begin{tabular}{|c|c|c|c|c|}
\hline \multicolumn{5}{|l|}{ Estado civil } \\
\hline Com companheiro & 64 & $30(46,9)$ & $34(53,1)$ & \multirow[t]{2}{*}{0,4202} \\
\hline Sem companheiro & 37 & $22(59,5)$ & $15(40,5)$ & \\
\hline \multicolumn{5}{|l|}{ Filhos } \\
\hline Nenhum & 36 & $22(61,1)$ & $14(38,9)$ & \multirow[t]{3}{*}{0,3541} \\
\hline Um & 28 & $13(46,4)$ & $15(53,6)$ & \\
\hline Dois ou mais & 37 & $17(45,9)$ & $20(54,1)$ & \\
\hline \multicolumn{5}{|l|}{ Categoria Profissional } \\
\hline Teleatendente & 16 & $8(50)$ & $8(50)$ & \multirow[t]{5}{*}{0,3652} \\
\hline Condutor Socorrista & 22 & $9(40,9)$ & $13(59,1)$ & \\
\hline Auxiliar de Enf. & 14 & $6(42,9)$ & $8(57,1)$ & \\
\hline Enfermeiro & 19 & $9(47,4)$ & $10(52,6)$ & \\
\hline Médico & 30 & $20(66,7)$ & $10(33,3)$ & \\
\hline \multicolumn{5}{|l|}{ Turno de Trabalho } \\
\hline Dia & 44 & $30(68,2)$ & $14(31,8)$ & \multirow[t]{3}{*}{$0,0075^{\star}$} \\
\hline Dia e Noite & 21 & $10(47,6)$ & $11(52,4)$ & \\
\hline Noite & 36 & $12(33,3)$ & $24(66,7)$ & \\
\hline \multicolumn{5}{|l|}{ Outro vínculo } \\
\hline Sim & 61 & $34(57,4)$ & $17(42,5)$ & \multirow[t]{2}{*}{0,0563} \\
\hline Não & 40 & $26(42,6)$ & $23(57,5)$ & \\
\hline \multicolumn{5}{|l|}{ Renda Familiar } \\
\hline 1 a 5 Salários & 45 & $23(51,1)$ & $22(48,9$ & \multirow[t]{3}{*}{0,4169} \\
\hline 6 a 10 Salários & 26 & $11(42,3)$ & $15(57,7)$ & \\
\hline Acima de 10 Salários & 30 & $18(60)$ & $12(40)$ & \\
\hline
\end{tabular}

Teste Wilcoxon

*Significativo ao nível de confiança de 95\%

Observa-se na Tabela 1 que mais da metade dos participantes tinha idade inferior a 40 anos, era do sexo masculino, possuía companheiro e tinha filhos. Em relação às características profissionais, mais da metade tinha mais de um vínculo empregatício e parcela significativa atuava no SAMU durante o dia e tinham renda de um a cinco salários mínimos.

Observa-se que 52 profissionais $(51,9 \%)$ apresentaram risco para desenvolvimento e manifestação da síndrome de Burnout - caracterizada por pontuação elevada para exaustão emocional e despersonalização e baixa pontuação na realização pessoal. Este risco foi mais frequente em indivíduos do sexo feminino, sem companheiro, sem filhos e com até 40 anos.

No que se refere às condições de trabalho, foi mais frequente entre aqueles que trabalhavam durante o dia, tinham outro vínculo empregatício e renda maior do que 10 salários mínimos. Já em relação à categoria profissional, maior proporção de risco foi observada entre os médicos, seguido pelos teleatendentes, enfermeiros, condutores e auxiliares de enfermagem. Contudo, a diferença só foi significativa em relação ao turno de trabalho - maior entre os profissionais que trabalhavam durante o dia $\left(p=0,0075^{\star}\right)$. 
Em relação aos domínios do instrumento que avaliou o risco para Síndrome de Burnout, observa-se, na Tabela 2, que todos os profissionais apresentaram baixa Realização pessoal, visto que a pontuação variou entre 10 a 17 pontos; $84(85,1 \%)$ profissionais apresentaram elevada Despersonalização - pontuação entre 18 e 22 pontos; e $55(56,4 \%)$ apresentaram baixa Exaustão profissional - pontuação entre 1 a 9 pontos.

Tabela 2 - Escore médio do MBI segundo variáveis sócio-demográficas e profissionais. Maringá, PR, Brasil, 2017

\section{VARIÁVEIS}

\begin{tabular}{cccccc}
\multicolumn{2}{c}{ Exaustão emocional } & \multicolumn{2}{c}{ Realização pessoal } & \multicolumn{2}{c}{ Despersonalização } \\
\hline $\begin{array}{l}\text { Escore } \\
\text { Médio }\end{array}$ & p-valor & $\begin{array}{l}\text { Escore } \\
\text { Médio }\end{array}$ & p-valor & $\begin{array}{l}\text { Escore } \\
\text { Médio }\end{array}$ & p-valor \\
\hline
\end{tabular}

\begin{tabular}{|c|c|c|c|c|c|c|}
\hline Sexo & & & & & & \\
\hline Masculino & 47,9 & \multirow[t]{2}{*}{0,1202} & 45,5 & \multirow[t]{2}{*}{$0,0429 *$} & 47,6 & \multirow[t]{2}{*}{0,1410} \\
\hline Feminino & 54,7 & & 55,6 & & 53,8 & \\
\hline \multicolumn{7}{|l|}{ Faixa Etária } \\
\hline Menos de 40 anos & 52,4 & \multirow[t]{2}{*}{0,0940} & 47,5 & \multirow[t]{2}{*}{0,9191} & 45,1 & \multirow[t]{2}{*}{$0,0221^{\star}$} \\
\hline 40 anos ou mais & 44,5 & & 53 & & 57 & \\
\hline \multicolumn{7}{|l|}{ Estado Civil } \\
\hline Com companheiro & 51,1 & \multirow[t]{2}{*}{0,4889} & 53,4 & \multirow[t]{2}{*}{0,1583} & 53,5 & \multirow[t]{2}{*}{0,1429} \\
\hline Sem companheiro & 50,9 & & 47,4 & & 47,2 & \\
\hline \multicolumn{7}{|l|}{ Número de filhos } \\
\hline Nenhum & 58,7 & \multirow[t]{3}{*}{0,1409} & 48,7 & \multirow[t]{3}{*}{0,5229} & 49,3 & \multirow[t]{3}{*}{0,4907} \\
\hline Um & 45,8 & & 48,2 & & 56,5 & \\
\hline Dois ou mais & 47,5 & & 55,3 & & 48,4 & \\
\hline \multicolumn{7}{|l|}{ Categoria Profissional } \\
\hline Teleatendente & 58 & \multirow[t]{5}{*}{0,2267} & 63 & \multirow[t]{5}{*}{0,3729} & 38,9 & \multirow[t]{5}{*}{0,2593} \\
\hline Condutor Socorrista & 50,8 & & 49,6 & & 44,9 & \\
\hline Auxiliar de Enf. & 39,2 & & 51,3 & & 58,3 & \\
\hline Enfermeiro & 49,9 & & 42,8 & & 51,2 & \\
\hline Médico & 56,7 & & 50,1 & & 54,8 & \\
\hline \multicolumn{7}{|l|}{ Turno de Trabalho } \\
\hline Dia & 59,8 & \multirow[t]{3}{*}{$0,0212^{\star}$} & 53,9 & \multirow[t]{3}{*}{0,3224} & 51,4 & \multirow[t]{3}{*}{0,8684} \\
\hline Dia e Noite & 41,9 & & 54,9 & & 53,3 & \\
\hline Noite & 48 & & 45,1 & & 49,1 & \\
\hline \multicolumn{7}{|l|}{ Outro Vínculo } \\
\hline Sim & 51,1 & \multirow[t]{2}{*}{0,4889} & 53,4 & \multirow[t]{2}{*}{0,1583} & 53,5 & \multirow[t]{2}{*}{0,1429} \\
\hline Não & 50,9 & & 47,4 & & 47,2 & \\
\hline \multicolumn{7}{|l|}{ Renda Familiar } \\
\hline 1 a 5 Salários & 54,1 & \multirow[t]{3}{*}{0,7220} & 52,2 & \multirow[t]{3}{*}{0,6960} & 50,4 & \multirow[t]{3}{*}{0,8544} \\
\hline 6 a 10 Salários & 47,7 & & 53,2 & & 49,3 & \\
\hline Mais de 10 Salários & 50,8 & & 47,2 & & 53,4 & \\
\hline
\end{tabular}

*Significativo ao nível de confiança de $95 \%$ 
Considerando os escores encontrados para cada uma das variáveis em estudo, nas três dimensões que compõem a Síndrome de Burnout, observa-se, na Tabela 2, diferença significativa entre os sexos em relação à Realização pessoal $(p=0,0429 *)$, sendo que as mulheres apresentam maior escore médio. Entretanto, elas apresentaram um escore médio maior em relação aos demais domínios, ou seja, estão mais exaustas emocionalmente e mais despersonalizadas.

Com relação à faixa etária, os profissionais com 40 anos ou mais apresentaram risco significativamente maior $(p=0,0221)$ para Despersonalização e maior escore médio para Realização pessoal (53). Em contrapartida, foram os mais jovens que apresentaram maior Exaustão emocional $(52,4)$.

No que se refere à categoria profissional, os teleatendentes $(58)$ e os médicos $(56,7)$ foram os que apresentaram maiores escores médios na dimensão Exaustão emocional. Já em relação à dimensão Realização pessoal, os teleatendentes apresentaram os maiores escores médios $(63,0)$, enquanto os enfermeiros, os menores $(42,8)$. Quanto à dimensão Despersonalização, os auxiliares de enfermagem apresentaram os maiores escores médios $(58,3)$, seguidos pelos médicos $(54,8)$ e enfermeiros $(51,2)$.

Os profissionais que trabalhavam durante o período diurno apresentaram maior Exaustão emocional $(59,8)$ quando comparando com os que trabalhavam no período noturno $(48,0)$, ou que cumpriam escalas durante dia e noite $(41,9)$, com diferença significativa $\left(p=0,0212^{\star}\right)$. Os participantes que atuavam no período noturno também apresentaram escore médio menor na dimensão Realização pessoal $(45,1)$ e na dimensão Despersonalização $(49,1)$.

Em relação à existência de outro vínculo empregatício, apesar de não ter sido encontrada diferença significativa, os profissionais que tinham mais de um vínculo apresentaram maior Exaustão emocional $(51,1)$ e Despersonalização $(53,5)$, embora também apresentassem escore indicativo de maior Realização pessoal $(53,4)$.

\section{DISCUSSÃO}

Os achados deste estudo apontam que os profissionais que atuam no SAMU estão sujeitos ao desenvolvimento da Síndrome de Burnout, o que provavelmente esteja relacionado ao fato de serem constantemente expostos a situações de estresse e pressão. Contudo, de acordo com os resultados, é possível inferir que alguns profissionais são mais resilientes do que outros e que algumas características contribuem para isto.

Quando se considera o sexo dos indivíduos, constata-se que apesar de não ter sido observada diferença significativa, neste estudo as mulheres apresentaram maior risco para desenvolver a Síndrome de Burnout. Este aspecto pode estar relacionado à associação com outros fatores, como a dupla jornada, em que muitas vezes elas conciliam as atividades do trabalho com as atividades familiares. Assim como esta pesquisa, estudos apontam que as mulheres apresentam maior risco de manifestar a síndrome em decorrência, especialmente, da "Exaustão emocional", uma vez que apresentam características próprias do gênero(5,9-10).

Os estudos ainda são controversos sobre a relação entre estado civil e risco de síndrome de Burnout. Neste, o risco de manifestar a síndrome foi maior entre os profissionais sem companheiros e sem filhos, o que reforça resultados de estudos que apontam que os solteiros sofrem em demasia pela ausência de laços afetivos e de um relacionamento familiar que represente estabilidade e companheirismo, o que os torna mais vulneráveis à síndrome de Burnout ${ }^{(8,11)}$.

Estudo realizado com técnicos de enfermagem concluiu que o fato de ser solteiro gera descompromisso com a responsabilidade familiar, o que pode constituir fator protetor à manifestação da síndrome de Burnout. ${ }^{(4)}$ Por outro lado, pessoas com laços familiares 
em união estável estão mais preparadas emocionalmente para enfrentar problemas, sendo vulneráveis apenas à "Exaustão emocional" e não a manifestação da síndrome de Burnout ${ }^{(4,12-13)}$. Entretanto, não basta ter um relacionamento, este precisa ser "bom", já que a presença de um companheiro também pode constituir fator estressor, interferindo na carreira pessoal e profissional do indivíduo. Deste modo, em indivíduos solteiros, com elevada autoestima, o risco de manifestar a síndrome de Burnout é minimizado(8,11).

Quanto à idade, constatou-se que, embora o risco para o desenvolvimento da síndrome de Burnouttenha sido mais frequente entre os profissionais com até 40 anos, foram os mais velhos que apresentaram risco significativamente maior para Despersonalização $\left(p=0,0221^{\star}\right)$. É importante considerar que atrelada à idade está o tempo de atuação no serviço. Neste sentido, diferentes aspectos, a exemplo da mecanização da assistência, podem potencializar a deseprsonalização, uma vez que o convívio com colegas vai se tornando rotineiro, modificando a maneira como os profissionais lidam com os clientes, chefias e colegas de trabalho ${ }^{(1,10)}$.

Ainda em relação à idade, ressalta-se que a Exaustão emocional foi a dimensão menos comprometida entre os profissionais com mais de 40 anos. Acredita-se que isto se deve ao fato de a mesma ter efeito protetor para o processo de desenvolvimento da síndrome de Burnout, uma espécie de bônus para os anos de trabalho, já que é com a experiência profissional que o domínio técnico e científico é adquirido, e por consequência maior segurança/autoconfiança(1).

O risco de manifestar Burnout foi mais frequente entre os profissionais que trabalhavam durante o dia, o que pode ser explicado pelo fato de ser o período de maior fluxo de ocorrências e, por conseguinte, de maior exposição a situações estressantes. Também pode interferir neste resultado o fato de durante o dia atuarem no serviço um maior número de profissionais, visto que o relacionamento interpessoal e convívio com os demais membros da equipe multiprofissional podem exercer influência, em especial no que tange à Exaustão emocional(4).

Em relação à categoria profissional, o risco para desenvolver o Burnout foi mais frequente entre os médicos e os teleatendentes. Este resultado pode estar associado ao perfil profissional destas categorias. Por exemplo, os médicos vivenciam a pressão diária da assistência móvel e da regulação do SAMU, atividade igualmente estressante associada a longas jornadas de trabalho, pois é comum terem dois ou mais vínculos empregatícios e estes fatores podem contribuir para maior Despersonalização e Exaustão emocional.

Os teleatendentes, por sua vez, também estão vulneráveis ao risco de manifestação de Burnout, principalmente em decorrência da Exaustão emocional. Isso por que, mesmo sem manter contato direto com o paciente, oferecem atendimento via telefone, o que os impossibilita de serem mais resolutivos ${ }^{(1,5,11)}$.

Apesar de profissionais com formação superior serem mais realizados pessoal e profissionalmente, experimentam maiores exigências e responsabilidades no processo de trabalho, o que pode gerar condições estressantes. Em contrapartida, as categorias que não exigem formação superior especificam a realização pessoal é menor. Ademais, todos os profissionais vivenciam exigências no ambiente de trabalho, assim como estão expostos ao convívio com a equipe e aos relacionamentos interpessoais ${ }^{(13,14)}$.

Sendo assim, as condições de trabalho e o clima em equipe devem ser consideradas nos serviços de saúde, uma vez que o risco de manifestar o Burnout somado à insatisfação profissional interferem na qualidade do trabalho, resultando em aumento do número de acidentes, iatrogenias, conflitos na equipe e falta de humanização na assistência ao paciente ${ }^{(1,5,11)}$.

Neste estudo, mais da metade dos profissionais possuía outro vínculo empregatício e, embora não tenha sido encontrada diferença significativa, a proporção para o risco foi maior entre eles. Profissionais que trabalham em outro emprego possuem rotinas mais estressantes e maior tendência a Despersonalização, devido ao aumento da sobrecarga, 
do número de horas de trabalho, e do tempo destinado ao atendimento a pacientes. Além disso, o profissional precisa lidar com diferentes rotinas, culturas organizacionais, chefia e outro perfil de equipe de trabalho, o que pode interferir em sua atuação e no rendimento de seu trabalho(5).

O vínculo com outra empresa exige mais do profissional, pois muitas vezes, diuturnamente, desempenha atividades diferentes e isto aumenta o risco de manifestar a síndrome de Burnout ${ }^{(5,13)}$.

A faixa salarial também parece influenciar o risco em desenvolver a síndrome de Burnout, e isto ocorreu tanto entre aqueles que possuíam renda inferior a cinco salários mínimos, quanto entre os com mais de 10 salários mínimos. Entre os primeiros, é provável que isto ocorra porque eles vivenciam a tensão desencadeada por uma renda insuficiente para suas necessidades, e entre os com renda maior, porque provavelmente esta renda é fruto de mais de um vínculo empregatício e todos os problemas deles decorrentes ${ }^{(13)}$.

Possíveis limitações do estudo relacionam-se ao fato de ter sido realizado em apenas um serviço e de sua amostra não ter sido selecionada de forma aleatória. De qualquer modo, seus resultados permitiram identificar aspectos importantes sobre a temática e recomendar que estudos futuros utilizem abordagem qualitativa a fim de explorar, com maior profundidade, como e em que condições a Síndrome Burnout se manifesta nesta população.

\section{CONCLUSÃO}

Os resultados deste estudo apontam que mais da metade dos participantes apresentaram risco para o desenvolvimento do Burnout, com destaque para os profissionais médicos e teleatendentes. Além disso, os trabalhadores com até 40 anos, que trabalhavam somente durante o dia e que possuíam outro vínculo empregatício apresentam mais chances de manifestarem a síndrome. Desta maneira, pode-se concluir que o risco para o desenvolvimento do Burnout atinge cinco a cada dez profissionais que atuam no Serviço de Atendimento Móvel de Urgência.

Estes resultados podem subsidiar a implementação de ações voltadas para a melhoria das condições de trabalho e da relação entre os profissionais que atuam em serviços de saúde como o SAMU. Devem ser incluídas estratégias que possam minimizar os efeitos do trabalho na qualidade de vida dos profissionais, tais como atividades ocupacionais e de alívio do estresse inerentes ao ambiente, a fim de preservar a saúde destes trabalhadores.

\section{REFERÊNCIAS}

1. Martins LF, Laport TJ, Menezes V de P, Medeiros PB, Ronzani TM. Esgotamento entre profissionais da Atenção Primaria à Saúde. Cienc. saude colet. [Internet]. 2014 [acesso em 02 abr 2017]; 19(12). Disponível em: http://dx.doi.org/10.1590/1413-812320141912.03202013.

2. Pêgo FPL e, Pêgo DR. Síndrome de Burnout. Rev bras med trab. [Internet]. 2016 [acesso em 02 abr 2017]; 14(2). Disponível em: http://dx.doi.org/10.5327/Z1679-443520162215.

3. Trigo TR. Validade fatorial do Maslach Burnout Inventory-Human Services Survey (MBI-HSS) em uma amostra brasileira de auxiliares de enfermagem de um hospital universitário: influência da depressão[dissertação]. São Paulo (SP): Faculdade de Medicina da Universidade de São Paulo; 2011.

4. Ferreira NN, Lucca SR de. Síndrome de Burnout em Técnicos de Enfermagem de um hospital público do Estado de São Paulo. Rev. bras. epidemiol. [Internet]. 2015 [acesso em 02 abr 2017]; 18(1). Disponível em: http://dx.doi.org/10.1590/1980-5497201500010006. 
5. Cornelius A, Carlotto MS. Síndrome de Burnout em profissionais de Atendimento de Urgência. Rev Psicol. Foco [Internet]. 2007 [acesso em 02 abr 2017]; 1(1). Disponível em: http://revistas.fw.uri.br/index. php/psicologiaemfoco/article/view/1125.

6. Ministério da Saúde (BR). Política nacional de atenção às urgências. [Internet] Brasília: Ministério da Saúde; 2006 [acesso em 21 out 2017]. Disponível em: http://bvsms.saude.gov.br/bvs/publicacoes/ politica nacional atencao urgencias 3ed.pdf.

7. Cabral AP da S, Souza WV de, Lima MLC de. Serviço de Atendimento Móvel de Urgência: um observatório dos acidentes de transporte terrestre em nível local. Rev. bras. epidemiol. [Internet]. 2011 [acesso em 21 out 2017]; 1(14). Disponível em: http://dx.doi.org/10.1590/S1415-790X2011000100001.

8. Stumm EMF, Ribeiro G, Kirchner RM, Loro MM, Rosanelli CLSP. Avaliação da saúde e qualidade de vida: profissionais de um SAMU. Cogitare enferm [Internet]. 2009 [acesso em 21 out 2017]; 4(14). Disponível em: http://dx.doi.org/10.5380/ce.v14i4.16374.

9. Soares INL, Souza LCG de, Castro AFL, Alves CF de O. Análise do estresse ocupacional e da síndrome de Burnout em profissionais da estratégia saúde da família no município de Maceió/AL. Semente [Internet]. 2011 [acesso em 21 out 2017]; 6(6). Disponível em: http://revistas.cesmac.edu.br/index.php/ semente/article/view/147.

10. Zanatta $A B$, Lucca SR de. Prevalence of Burnout syndrome in health professionals of an oncohematological pediatric hospital. Rev. Esc. Enferm. USP. [Internet]. 2015 [acesso em 21 out 2017]; 49(2). Disponível em: https://doi.org/10.1590/S0080-623420150000200010.

11. Carvalho D de, Silva N, Bachur JA, Mesquita JLF, França-Botelho AC. Síndrome de Burnout em profissionais da área da saúde atuantes em dois municípios do interior de Minas Gerais - Brasil.

Rev. Contexto Saúde [Internet]. 2016 [acesso em 21 out 2017]; 16(31). Disponível em: http://dx.doi. org/10.21527/2176-7114.2016.31.139-148.

12. Murassaki ACY, Versa GLG da S, Inoue KC, Melo WA de, Matsuda LM. Estresse em enfermeiros intensivistas e a condição chefe/não chefe de família. Ciênc. cuid. saúde. [Internet]. 2011 [acesso em 21 out 2017]; 10(4). Disponível em: http://dx.doi.org/10.4025/cienccuidsaude.v10i4.18320.

13. Luz LM, Torres RRB, Sarmento KMV de Q, Sales JMR, Farias KN, Marques MB. Síndrome de Burnout em profissionais de serviço de atendimento móvel de urgência. J. res. fundam. care. [Internet]. 2017 [acesso em 21 out 2017]; 1(9). Disponível em: http://dx.doi.org/10.9789/2175-5361.2017.v9i1.238-246.

14. Fernandes MA, Souza FK de, Santos JS dos, Rodrigues J de A, Marziale HP. Síndrome de Burnout em profissionais de enfermagem do serviço de atendimento móvel de urgência. J. res. fundam. care. [Internet]. 2012 [acesso em 21 out 2017]; 4(4). Disponível em: https://www.redalyc.org/ html/5057/505750895003/.

Recebido: 30/09/2018

Finalizado: 03/05/2019

Autor Correspondente:

Gabriela Bettoni Figueiroa

Pontifícia Católica de Campinas

Av. José Pancetti, 861 - 13033740 - Campinas, SP, Brasil

E-mail: bybettoni@hotmail.com

Contribuição dos autores:

Contribuições substanciais para a concepção ou desenho do estudo; ou a aquisição, análise ou interpretação de dados do estudo - GBF, HEP, IRB, USS

Elaboração e revisão crítica do conteúdo intelectual do estudo - GBF, HEP, NLMG, SSM 
Aprovação da versão final do estudo a ser publicado - GBF, NLMG, SSM

Responsável por todos os aspectos do estudo, assegurando as questões de precisão ou integridade de qualquer parte do estudo - GBF 